University of Nebraska - Lincoln

DigitalCommons@University of Nebraska - Lincoln

Papers in Analytical Chemistry

Chemical and Biomolecular Engineering

Research and Publications

$11-1-2004$

Exergy use in bioenergetics

Yasar Demirel

Virginia Polytechnic Institute and State University, ydemirel2@unl.edu

Follow this and additional works at: https://digitalcommons.unl.edu/chemenganalytical

Part of the Chemical Engineering Commons

Demirel, Yasar, "Exergy use in bioenergetics" (2004). Papers in Analytical Chemistry. 4.

https://digitalcommons.unl.edu/chemenganalytical/4

This Article is brought to you for free and open access by the Chemical and Biomolecular Engineering Research and Publications at DigitalCommons@University of Nebraska - Lincoln. It has been accepted for inclusion in Papers in Analytical Chemistry by an authorized administrator of DigitalCommons@University of Nebraska - Lincoln. 


\title{
Exergy use in bioenergetics
}

\begin{abstract}
Every developed and adapted biological system extracts useful energy from outside, converts, stores it, and uses for muscular contraction, substrate transport, protein synthesis, and other energy utilizing processes. This energy management in a living cell is called the bioenergetics, and the useful energy is the exergy, which is destroyed in every irreversible process because of the entropy production. The converted exergy is the adenosine triphosphate (ATP) produced through the oxidative phosphorylation coupled to respiration in which the exergy originates from oxidation of reducing equivalents of nutrients. A living cell uses the ATP for all the energy demanding activities; it has to maintain nonvanishing thermodynamic forces, such as electrochemical potential gradient, and hence is an open, nonequilibrium system, which manages the exergy destruction and power production to adapt the fluctuations in energy demand and production within mitochondria. A simplified example presented here shows that the use of exergy analysis is helpful for understanding and analysing oxidative phosphorylation in bioenergetics.
\end{abstract}

Keywords: degree of coupling; dissipation; exergy; nonequilibrium thermodynamics; oxidative phosphorylation.

Comments: This paper originally was published in The Journal of "Int. J. Exergy", Vol.1, No.1, 2004 and Copyright C 2004 Inderscience Enterprises Ltd. 


\section{Introduction}

Exergy is mainly associated with the energy interactions between a physical process and the environment. As a process progress towards equilibrium with the environment, we explore the ways of extracting the maximum useful shaft work or electrical work. This naturally implies the exergy-aided cost optimization based on the combination of the thermodynamic optimum and the principles of economic engineering. However, the implications of exergy use in biological systems are addressed inadequately, although a living cell is an open system with continuous energy and matter interactions with the environment, and operating away from equilibrium as an organized and dissipative structure.

Mitochondria in a living cell contain the inner and outer membranes made of bilayers, which may influence the coupling and local gradients of ions, molecules, and macromolecules in the regulation of energy metabolism (Mannella, 2000). Structural anisotropy in the mitochondria is necessary for a chemical reaction to be coupled with a substrate flow process in a cell according to the Curie-Prigogine principle (Demirel and Sandler, 2002). The inner membrane houses the respiratory chain and ATP synthesis in oxidative phosphorylation (OP). The energy source for the phosphorylation is the oxidation of reducing equivalents of nutrients via the respiratory chain. Mitochondria have various complexes for converting substrate into energy (Lemasters, 1984). These complexes pump protons $(\mathrm{H}+)$ from the matrix into the intermembrane space (Kadenbach et al., 2000). This forms an electrochemical proton gradient across the inner mitochondrial membrane. The ATP synthase uses this electrochemical potential gradient for the synthesis of ATP from adenosine diphosphate (ADP) and phosphate (Pi), which drives the majority of energy utilizing processes in the cell. The energy metabolism in the cell has to optimize the rate of energy production and constantly changing energy demand, such as increasing thermogenesis at low temperatures, preparing appropriate responses under stress conditions, and efficient use of nutrients. TIus optimization incorporates several control mechanisms including a slip of proton pumps and proton leak across the bilayer (Kadenbach et al., 2000). However mitochondria cannot maximize the ATP production and exergetic efficiency at the same time (Cairns et al., 1998). This study presents the exergetic aspects in bioenergetics using a simplified example of OP.

\section{Nonequilibrium thermodynamics}

In a nonequilibrium system some of the intensive parameters are functions of time and position and a local thermodynamic equilibrium state exists in small volume elements at each point. These volume elements contain enough molecules to be treated as homogeneous, and the thermodynamic properties may be determined at every point in the same way as for substances in equilibrium. The postulate of local thermodynamic equilibrium is valid if the gradients of intensive thermodynamic functions are small, and their local values vary slowly in comparison with the relaxation time of the local state of the system (Cairns et al., 1998; Demirel, 2002; Katchalsky and Curran, 1967; Kondepudi and Prigogine, 1999). The local thermodynamic equilibrium implies the microscopic reversibility that is the probability of a microscopic process occurring in one direction of time is the same as that in the reverse direction (Kondepudi and Prigogine, 1999). For a simple reversible reaction, the principle of microscopic reversibility means that if one of the paths is preferred for the forward reaction, the same path must also be preferred for the reverse reaction (Levenspiel, 1999):

Using the local thermodynamic equilibrium approach, nonequilibrium thermodynamics calculates the rate of entropy production $(d i S / d t)$ or the dissipation $\psi$ due to irreversible processes in a system. The dissipation function $\psi$ is derived from the entropy balance beside the general balance equations of mass, momentum, energy, and the Gibbs relation, and expressed by 


$$
\Psi=T\left(\frac{d_{i} S}{d t}\right)=\sum_{k=1}^{m} J_{k} X_{k}
$$

where $J_{k}$ is the flow, or output, such as energy flow, or rate of reaction, and $X_{k}$ is the thermodynamic force that causes a flow, such as chemical potential gradient or chemical affinity $\mathrm{A}, \mathrm{A}_{\mathrm{j}},=-\Sigma A_{j} v_{i j}$, where $v_{i j}$ is the stoichiometric coefficients of reaction $j$. Equation (1) identifies the conjugate flows and forces to be used in the phenomenological equations, which relate the forces and flows linearly through the phenomenological coefficients $L_{i j}$

$$
J_{i}=\sum_{i=1}^{m} L_{i k} X_{k} \quad(i, k=1,2, \ldots, m) .
$$

In Equation (2), the cross coefficients $L_{i j}(\mathrm{i} \# j)$ are symmetric according to the Onsager reciprocal relations, and relate the coupled flows. Coupling implies an interrelation between flow $i$ and flow $j$ so that, for example, a substance can flow without or against its conjugate driving force (Caplan and Essig, 1983; Demirel, 2002; Howland and Needleman, 2000; Jencks, 1989; Stucki et al., 1983;) without violating the second law of thermodynamics.

\section{Energy conversion}

Experiments and empirical analysis of different cellular process show that linear relations exist between the rate of respiration and growth of many microbial systems with mechanisms to optimize the exergetic efficiency for some of the individual processes of OP (Lemasters, 1984; Mannella, 2000; Rigoulet et al., 1998; Rottenberg, 1979; Santillan et al., 1997; Soboll and Stucki, 1985; Stucki, 1980a, 1984, 1991; Stucki et al., 1983). This might be the result of a special kinetics of enzyme reaction, such as the Michaelis-Menten equation, which shows that the enzyme reactions in certain regions can be approximated as linear (Levenspiel, 1999). Existence of a multiple inflection point in the force-flow space of a system of enzyme-mediated reactions suggests that a steady state in the vicinity of the linear region occurs over a considerable range, and linear relations may be favored for a highly coupled energy transducer, since local asymptotic stability is guaranteed, although the system may be far from equilibrium (Baumann, 1991; Caplan and Essig, 1983; Natl , 1998; Nielsen, 1997; Pfeiffer et al., 2001; Rothschild et al., 1980; Rottenberg, 1979; Stucki, 1980a, 1984; Walz and Caplan, 1995; Westerhoff, 1982). Intrinsic linearity would have an energetic advantage as a consequence of evolution (Heinrich and Schuster, 1998; Rothschild et al., 1980; Stucki et al., 1983). Stucki (1980a) demonstrated that variation of the phosphate potential at constant oxidation potential yields linear flow-force relationships in the mitochondria. Extensive ranges of linearity are also reported for the reaction driving the active sodium transport in epithelial membranes, where the sodium transport occurs close to a stationary state (Candia and Reinach, 1982; Lagarde, 1976; Stucki et al., 1983) where the entropy production or the destruction of exergy reaches a minimum value. Cells optimize their free energy production and utilization by lowering their entropy production and hence exergy destruction (Toussaint and Schneider, 1998).

The second law of thermodynamics states that entropy production or exergy destruction as a consequence of the irreversibility is always positive. In mitochondria, a representative overall dissipation function or the exergy destruction for OP may be obtained from Equation (I), and expressed by (Caplan and Essig, 1983; Demirel, 2002; Katchalsky and Curran, 1967; Stucki, 1980a). 


$$
\Psi=J_{o} X_{o}+J_{p} X_{p} \geq 0
$$

where the input force $X_{0}$ is the redox potential of oxidisable substrates, and $X_{p}$ is the output force representing the affinity $\mathrm{A}$, or the phosphate potential expressed by $X_{p}=\left(\Delta \mathrm{G}_{\mathrm{p}}{ }^{0}+\mathrm{RT}\right.$ $\ln \left([\mathrm{ATP}] /\left([\mathrm{ADP}]\left[\mathrm{P}_{\mathrm{i}}\right]\right)\right)$, where $\Delta \mathrm{G}_{\mathrm{p}}{ }^{0}$ is the Gibbs free energy at standard conditions. The associated input flow $J_{o}$ is 'the net oxygen consumption, and the outflow $J_{p}$ is the net rate of ATP production. For the sum to be positive in Equation (3), we can have either $J_{o} X_{o}>0, J_{p} X_{p}>0$, or $J_{p} X_{p}<0$, while the other is positive and large enough to compensate the negative effect of the first term. If the system is an energy converter, we have the ATP production $J_{p} X_{p}$ (driven process), coupled to the respiration $J_{o} X_{o}$ (driving process) (Bisch, 1993; Caplan and Essig, 1983; Krupka, 1998; Prigogine, 1967; Rottenberg, 1979; Rottengberg and Gutman, 1977; Waldeck et al., 1998; Walz, 1979). In the uncoupled respiration no gradients are formed, and the respiration is not controlled by the energy consumption in the cell. This may be the result of a modified energy-coupled respiratory chain (Skulachev, 1998). This sequence of coupling is controlled at switch points where the mobility, specificity and catalysis of the coupling protein can be altered is specific ways such as sifted equilibria or regulated rates of conversion between one protein state and another (Krupka, 1998). Prigogine (1967) showed that the total exergy destruction reaches a minimum in a stationary state, which is the stability criterion of a stationary state (Kondepudi and Prigogine, 1999). Optimal performance regimes of biological systems are associated with minimum entropy production.

The exergetic efficiency 77 is defined as the ratio of dissipations due to output and input powers in $\mathrm{OP}$, and from Equation (3) we have

$$
\eta=-\frac{J_{p} X_{p}}{J_{o} \bar{X}_{o}} .
$$

It is customary to use the following simplified, representative linear phenomenological equations for the overall OP (Cairns et a], 1998; Stucki, 1980a)

$$
\begin{aligned}
& J_{p}=L_{p} X_{p}+L_{p o} X_{o} \\
& J_{o}=L_{o p} X_{p}+L_{o} X_{o} .
\end{aligned}
$$

The matrix of the phenomenological coefficients must be positive definite; for example, for a two-flow system we have $L_{o}>0, L_{p}>0$ and $L_{o} L_{p}-L_{o p} L_{p o}>0$. The cross-phenomenological coefficient $L_{o p}$ represents the mechanism of coupling of this complex coupled process. $L_{o}$ shows the influence of substrate availability on oxygen consumption (flow), $L_{p}$ is the feedback of the phosphate potential on ATP production (flow). The cross-coupling coefficient $L_{o p}$ shows the phosphate influence on oxygen flow, while $L_{p o}$ shows the substrate dependency of ATP production. Experiments show that Onsager's reciprocal relations holds for OP, and we have $L_{o p}=L_{p o}$, (Caplan and Essig, 1983; Stucki, 1980a). Equations (5) and (6) are valid only in the vicinity of the equilibrium state (Stucki, 1980a; Stucki et al., 1983). Such linear energy converters are characterized by an optimal efficiency $\eta_{\text {opt }}$ (Caplan and Essig, 


$$
\eta_{\text {opt }}=\left(\frac{q}{1+\sqrt{1-q^{2}}}\right)^{2}
$$

where $\mathrm{q}$ is called the degree of coupling that is expressed in terms of the phenomenological coefficients $L_{i j}$ (Kedem and Caplan, 1965)

$q=\frac{L_{o p}}{\left(L_{p} L_{o}\right)^{1 / 2}}$.

Here $\mathrm{q}$ is a lump-sum degree of coupling for the various individual degrees of coupling of different processes of OP driven by the respiration. Absolute values of degree of coupling vary from zero for completely uncoupled systems to unity for completely coupled systems $O<|q|<I$.

By dividing Equation (5) by Equation (6), and by further dividing the numerator and denominator by $X_{o}\left(L_{o} L_{p}\right)^{1 / 2}$, we obtain

$$
\eta=j x=-\frac{x+q}{q+1 / x}
$$

where

$$
j=\frac{J_{p}}{J_{o} Z}, x=\frac{X_{p} Z}{X_{o}}, Z=\left(\frac{L_{p}}{L_{o}}\right)^{1 / 2} .
$$

Equation (9) shows the exergetic efficiency $\eta$ in terms of the force ratio $\mathrm{x}$ and the degree of coupling $q$. The term $\mathrm{Z}$ is called the phenomenological stoichiometry (Caplan and Essig, 1983). The ratio $J_{p} / J_{o}$ is the conventional phosphate to oxygen consumption ratio.

\section{Exergy destroyed}

For the OP described by Equations (5) and (6), the exergy destroyed can be obtained from Equation (3) in terms of the force ratio $\mathrm{x}$ and the degree of coupling $\mathrm{q}$, and given by

$$
\Psi=\left(x^{2}+2 q x+1\right) L_{0} X_{0}^{2} .
$$

Minimum exergy destruction or the minimum entropy production at the stationary state provides a general evolution criterion as well as a stability criterion (Cairns et al., 1998; Glansdorff and Prigogine, 197 1; Kondepudi alld Prigogine, 1999; Nicolis and Prigogine, 1977; Toussaint and Schneider, 1998). Entropy production rate decreases as $|q|$ increases, reaching an optimum value at which $|q|=1$ corresponding to the steady state at equilibrium.

There are two important steady states identified in the cell: (i) static head (sh), and (ii) level flow (If). At static head, where the ATP production is zero $J_{p}=0$, the Coupling between the respiratory chain and OP emphasizes to maintain a phosphate Potential $X_{p}$, which can be obtained from Equation (5) as $(X p)_{s h}=-$ $q X_{o} / Z$, and the Static head force ratio $x_{s h}$ becomes $x_{s h}=-q$. The oxygen flow $J_{o}$ at static head is obtained from Equations (5) and (6)

$$
\left(J_{o}\right)_{\text {sh }}=L_{o} X_{o}\left(1-q^{2}\right)
$$


Where $L_{o}$ may be interpreted as the phenomenological conductance coefficient of the respiratory chain. If an uncoupling agent, such as dinitrophenol (Cairns et al., 1998), is used the ATP production vanishes and hence $X_{p}=0$, then Equation (5) becomes

$$
\left(J_{o}\right)_{\mathrm{unc}}=L_{o} X_{o} \text {. }
$$

Combining Equations (11) and (12), we obtain

$$
\left(J_{o}\right)_{\mathrm{sh}}=\left(J_{o}\right)_{\mathrm{unc}}\left(1-q^{2}\right) .
$$

Using the experimentally attainable static head condition (state 4 in mitochondria) and the uncoupled oxygen flow $\left(J_{o}\right)_{u n c}$ we can determine the degree of coupling q.

$$
q=\left(1-\frac{\left(J_{o}\right)_{\mathrm{sh}}}{\left(J_{o}\right)_{\mathrm{unc}}}\right)^{1 / 2} .
$$

At constant $X_{o}$ Equation (10) yields the minimum value of exergy destroyed at $x=-q$

$$
(\Psi)_{\text {sh }}=(\Psi)_{\min }=\left(1-q^{2}\right) L_{o} X_{o}^{2} .
$$

The ratio of dissipations expressed in Equations (15) and (10) depends only on the force ratio and the degree of coupling, and becomes an exergy distribution ratio over the values of $\mathrm{x}$.

$$
\phi_{1}=\frac{\Psi_{\mathrm{sh}}}{\Psi}=\frac{1-q^{2}}{x^{2}+2 q x+1} .
$$

At level flow, the phosphate potential vanishes, and no net work is performed by the mitochondria, and the flow ratio becomes

$$
j_{\mathrm{rs}}=\frac{L_{p 0}}{L_{0}}=q Z .
$$

The combination of Equations (16) and (17) yields an expression for estimating the phenomenological stoichiometry $Z$ from measured $J_{p} / J_{o}=P / O$ ratios at level flow

$$
Z=\frac{P / O}{\sqrt{1-\left(J_{o}\right)_{\mathrm{sh}} /\left(J_{o}\right)_{\mathrm{uxyc}}}} .
$$

The efficiency expressed in Equation (9) is zero at both static head and level flow, due to vanishing power at these states. Between the static head and level flow efficiency passes through an optimum, which is given in Equation (7).

The force ratio at optimal efficiency is expressed by (Stucki, 1980a) 


$$
x_{\mathrm{opt}}=-\sqrt{\eta_{\mathrm{opt}}}=-\frac{q}{1+\sqrt{1-q^{2}}} .
$$

The rate of optimal efficiency of OP is not characterized by the exergy decrease (Stucki, 1980a), and the exergy destroyed at optimal efficiency is given by

$$
\Psi_{\mathrm{opt}}=\frac{\left(1-x^{2}\right)^{2}}{1+x^{2}} L_{o} X_{o}^{2}
$$

Ratio of dissipations $\psi_{\text {opt }}$ and $\psi$, given in Equations (20) and (10) respectively, shows the effect of optimal operation on the exergy destruction in terms of the force ratio and the degree of coupling only

$$
\phi_{2}=\frac{\Psi_{\mathrm{opt}}}{\Psi}=\frac{\left(1-x^{2}\right)^{2}}{\left(1+x^{2}\right)\left(x^{2}+2 q x+1\right)}
$$

For the optimal efficiency to occur at steady state, OP progress with a load causing certain dissipation. Such a load $J_{L}$ is an ATP utilizing process in the cell, such as the transport of substances. A load, which will make the steady state as the optimal efficiency state, can be identified through the total exergy destroyed $\psi_{\mathrm{c}}$

$$
\Psi_{c}=J_{p} X_{p}+J_{o} X_{o}+J_{L} X
$$

Here $J_{L}$ is the net rate of ATP consumed and $\mathrm{X}$ is the driving force. If we assume that ATP-utilizing process is driven by the phosphate potential $X_{p}$ and the $J_{L}$ is linearly related to $X_{p}$ then we have $J_{L}=L X_{p}$ .Here $L$ is a phenomenological conductance coefficient. The dissipation caused by the load is $J_{L} X_{p}=$

$L X_{p}{ }^{2}$ and the total exergy destroyed is expressed by

$\Psi_{c}=\left(x^{2}\left(1+\frac{L}{L_{p}}\right)+2 q x+1\right) L_{0} X_{o}^{2}$.

Using Equation (19), and from the extremum of Equation (23), Stucki (1980a) found the condition

$$
\frac{L}{L_{p}}=\beta \sqrt{1-q^{2}}
$$

and called the conductance matching of OP. Here, $\beta$ shows the percentage of the conductance matching. After combining Equations (23) and (24), we obtain

$$
\Psi_{c}=\left(x^{2}\left(1+\beta \sqrt{1-q^{2}}\right)+2 q x+1\right) L_{0} X_{0}^{2}
$$

The exergies destroyed at static head with conductance matching $\left(\psi_{\mathrm{c}}\right)_{\mathrm{h}}$ and at the state of optimal efficiency $\left(\psi_{\mathbf{c}}\right)_{\text {opt }}$ are expressed by Stucki $(1980$ a) as follows 


$$
\begin{aligned}
& \left(\Psi_{c}\right)_{\mathrm{sh}}=\sqrt{1-x^{2}}\left(x^{2}+\sqrt{1-x^{2}}\right) L_{o} X_{o}^{2} \\
& \left(\Psi_{c}\right)_{\mathrm{opt}}=\frac{1-x^{2}}{1+x^{2}} L_{o} X_{o}^{2} .
\end{aligned}
$$

It is now possible to estimate the ratios of exergies $\boldsymbol{\sigma}_{3}$ and $\boldsymbol{\sigma}_{4}$ in terms of the force ratio $\mathrm{x}$ and the degree of coupling q by using Equations (25)-(27)

$$
\begin{gathered}
\phi_{3}=\frac{\left(\Psi_{c}\right)_{\mathrm{sh}}}{\Psi_{c}}=\frac{\sqrt{1-x^{2}}\left(x^{2}+\sqrt{1-x^{2}}\right)}{x^{2}\left(1+\beta \sqrt{1-q^{2}}\right)+2 q x+1} \\
\phi_{4}=\frac{\left(\Psi_{c}\right)_{\mathrm{opt}}}{\Psi_{c}}=\frac{1-x^{2}}{\left(1+x^{2}\right)\left(x^{2}\left(1+\beta \sqrt{1-q^{2}}\right)+2 q x+1\right)} .
\end{gathered}
$$

Equations (28) and (29) are analogous to those Equations (16) and (21) and show the ratios of exergy destructions with and without the load.

Linear OP is interpreted as a result of some type of optimization that the efficiency of various biological converters at far from equilibrium is greatly improved by linear relations between flows and forces (Stucki et al., 1983). Comparisoll of energy conversions in linear and nonlinear regions requires a combination of thermodynamic and kinetic considerations to express the exergetic efficiencies of nonlinear $\eta_{\mathrm{n} l}$ and linear $\boldsymbol{\eta}_{l}$ modes

$$
\eta_{n l}=-\left(\frac{J_{p}}{J_{o}}\right) \frac{X_{p}}{X_{0}}, \text { and } \eta_{l}=-\left(\frac{J_{p}}{J_{o}}\right) \frac{X_{p}}{X_{o}}
$$

Donating $\gamma$ as a measure for the relative binding affinity of a substrate $\left(\mathrm{H}^{+}\right)$on the either side of the membrane, the following inequalities are obtained for $\gamma \geq 1$ (Stucki et al., 1983)

$$
\begin{aligned}
& \eta_{n l}>\eta_{l} \text { for } \zeta=\frac{1}{q} \\
& \eta_{n i}<n_{l} \text { for } \zeta=q
\end{aligned}
$$

where $\zeta$ is the reduced stoichiometry $(Z / n)$, which is subjected to kinetic limitation $(1 / q) \geq \zeta \geq q$, and $\boldsymbol{n}$ is the mechanistic stoichiometry. Inequalities (31) and (32) suggest that at a certain value of $\zeta$ the efficiencies of nonlinear and linear modes become equal to each other. This means that there exist values for $\zeta$ where the energy converter operates more efficiently. A fully coupled system is incompatible with optimal efficiency in biological energy converters operating at nonvanishing output flows. A ratio of efficiencies at linear and nonlinear modes

$$
\eta_{r}=\frac{\eta_{l}}{\eta_{n l}}
$$

is a measure for the efficiency gain in linear mode operation. The efficiency in linear mode depends on only q (Equation (7)), while the efficiency nonlinear mode depends on input force X, beside $q$. In the nonlinear region, the efficiency decreases at high values of input force, and the force ratio at optimum operation $\mathrm{x}_{\mathrm{opt}, \mathrm{nl}}$ is shifted towards level flow where $\mathrm{x}=0$ (Stucki et al., 1983). I11 OP the input force is the redox potential of the oxidisable substrates and the output force is the phosphate potential. If these two forces are balanced, the system operates close to reversible equilibrium. This means that the system would be completely coupled, and two processes would merge into a single process governed by a single force. Experiments show that in mitochondria $\mathrm{y}<1$, and input force is well above 50RT (Caplan and 
Essig, 1983;Lemasters, 1984; Stucki, 1980a). For a fully coupled system in nonlinear region of a single force, the phosphate potential $X p$ would be very small. However, a dissipative structure can only be maintained with a considerable $X_{p}$. On the other hand, in the linear mode of operation, optimum force ratio $x_{o p t}$ does not depend on input force (Equation (19)) (Soboll and Stucki, 1985; Stucki, 1980a; Stucki et al., 1983).

The gain ratio $\eta_{r}$ can be calculated at a reference force ratio, such as $x_{o p t}$, which is a natural steady-state force ratio of OP in the liver cell (Stucki et al., 1983). This is seen as the result of adaptation of OP to various metabolic conditions and also results from thermodynamic buffering of reactions catalyzed by enzymes (Stucki, 1980b). Experimentally observed linearity of the several energy converters operating far from equilibrium is due to enzymatic feedback regulation with an evolutionary drive towards higher efficiency (Howland and Needleman, 2000; Stucki, 1980b, 1984). W11en the conductance matching is fulfilled, energy conversion could be accomplished without any losses if $|q|=1$, corresponding to maximal power output. However, $|\mathrm{q}|=1$ implies that load conductance coefficient L in Equation (24) vanishes, which is of no interest for a living organism, since the absence of a load leads to equilibrium representing the dead state. Therefore a living organism requires $|\mathrm{q}|<1$. The particular value of degree of coupling depends on the nature of the output required from the energy converter. Stucki (1980a, b) outlined various output modes:

- For a maximal net rate of ATP flow at optimal efficiency $\left(J_{p}\right)_{o p t}$ the degree of coupling is estimated as $q_{f}=0.786$

- For an economic net ATP flow $\left(J_{P \eta}\right)_{\text {opt }}$ we have $q_{f}^{e c}-=0.953$

- For a maximal output power $\left(J_{p} X_{p}\right)_{o p t}$ at optimal efficiency, $q_{p}=0.91$

- For an economic net output power $\left(J_{p} X_{p} \eta\right)_{\text {opt }}$ we have $q_{p}{ }^{e c}=0.972$.

These experimental results show that the degree of coupling is not a constant but a variable subject to metabolic requirements and physiological organ roles (Cairns et al., 1998; Soboll and Stucki, 1985; Stucki, 1980a). For example, fatty acids decrease the degree of coupling, and function as uncouples. Uncoupling is not restricted to thermoregulation; some uncoupling activity is favorable for the performance of the metabolic and even the energy-conserving functions of cellular respiration (Shlachev, 1998; Stucki et al., 1984). Mitochondria could regulate the degrees of coupling of OP depending on the energy demand of the cell. For example OP of the fed rats operates very close to conductance matching, i.e. at the state of optimal efficiency at the economic degree of coupling (Cairns et al., 1998; Stucki, 1980a, b).

Figure 1 shows the change of efficiencies $\eta$, given in Equation (9), in terms of flow ratio $x$ between -1 and 0 , and for the particular degrees of coupling $q_{f}, q_{p}, q_{f}{ }^{\mathrm{ec}}$ and $q_{p}{ }^{e c}$. As Equation (7) shows, the optimum efficiency values are only dependent on the degrees of coupling, and increase with increasing values of $q$. The values of efficiency reach maximums changing between 0.2359 and 0.6195 corresponding to $q_{f}$ and $q_{p}{ }^{e c}$, respectively. 
Figure 1 The change of efficiencies $\eta$, given in Equation (9), in terms of flow ratio $x$ and for the degrees of coupling $q_{f}, q_{p}, q_{f}{ }^{\mathrm{ec}}$ and $q_{p}{ }^{e c}$.

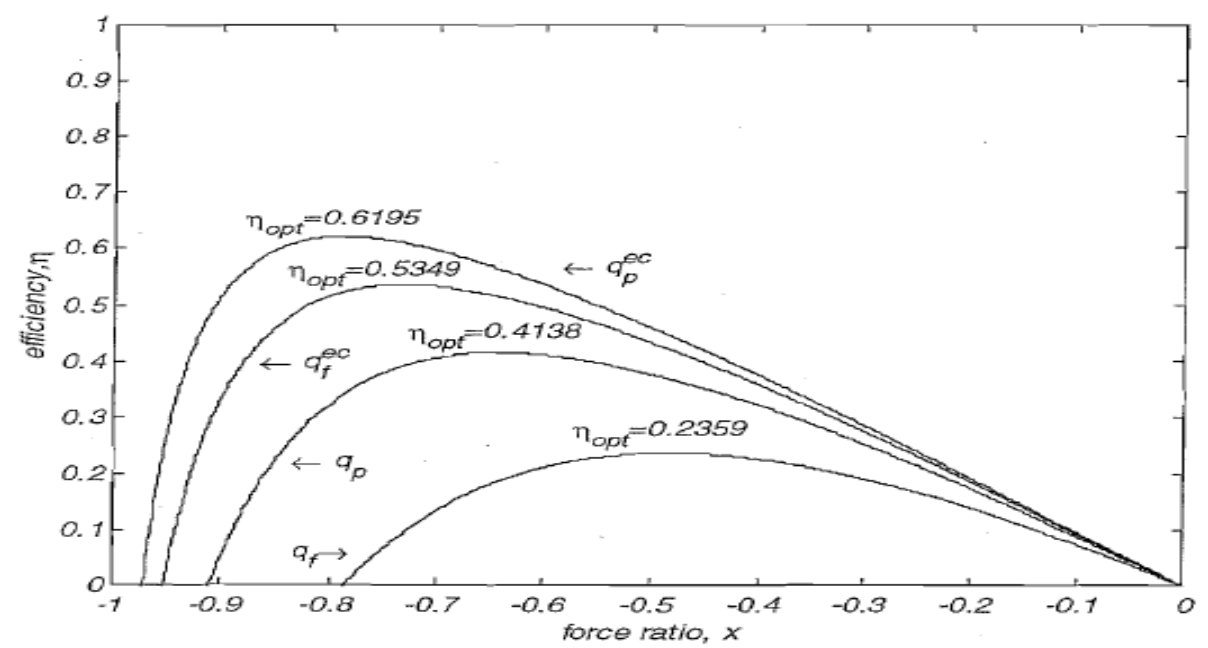

Figures 2-5 show the ratios of exergy destructions for unloaded and loaded OP in terms of $x$ and the four degrees of coupling $q_{f}, q_{p}, q_{f}{ }^{\mathrm{ec}}$ and $q_{p}{ }^{e c}$. These ratios change between $x=-1$ and $=0$ exhibiting a maximum value at a certain flow ratio $x$. Figure 2 shows that the values of $\boldsymbol{\sigma}_{1}$ reach unity at various values of $x$, and the economic degrees of coupling $q_{f}{ }^{\mathrm{ec}}$, and $q_{p}{ }^{e c}$ yield lower values of $\boldsymbol{\emptyset}_{1}$, compared with those obtained for $q_{f}, q_{p}$. Exergy destructions at static head are relatively lower at the degrees of coupling corresponding to economical ATP production and power output. Figure 3 shows that the values of $\mathrm{d} \boldsymbol{\sigma}_{2}$, expressed in Equation (21) with $x_{\text {opt }}=-\sqrt{ } \eta_{\text {opt }}$, reach some peak values higher than unity, and clearly shows that exergy destruction is not minimized at optimal efficiency of OP. The exergy destruction is the lowest at the degree of coupling corresponding to the economical power output. 
Figure 2 The change of ratio $\phi_{1}$, given in Equation (16), in terms of flow ratio $x$ and for the degrees of coupling $q_{f}, G_{p}, q_{f}^{\mathrm{mr}}$, and $q_{p}^{\mathrm{n}}$

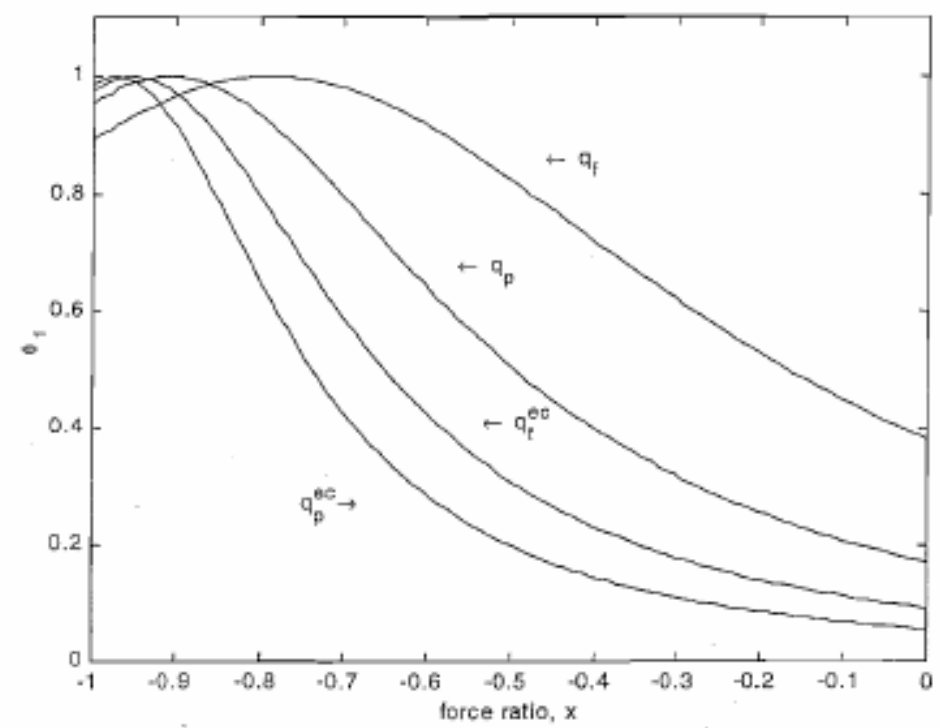

Figure 3 The change of ratio $\phi_{2}$, given in Equation (21), in terms of flow ratio $x$ and for the degrees of coupling $\mathscr{G}_{f} q_{p}, q_{f}^{t c}$, and $q_{p}^{s t}$

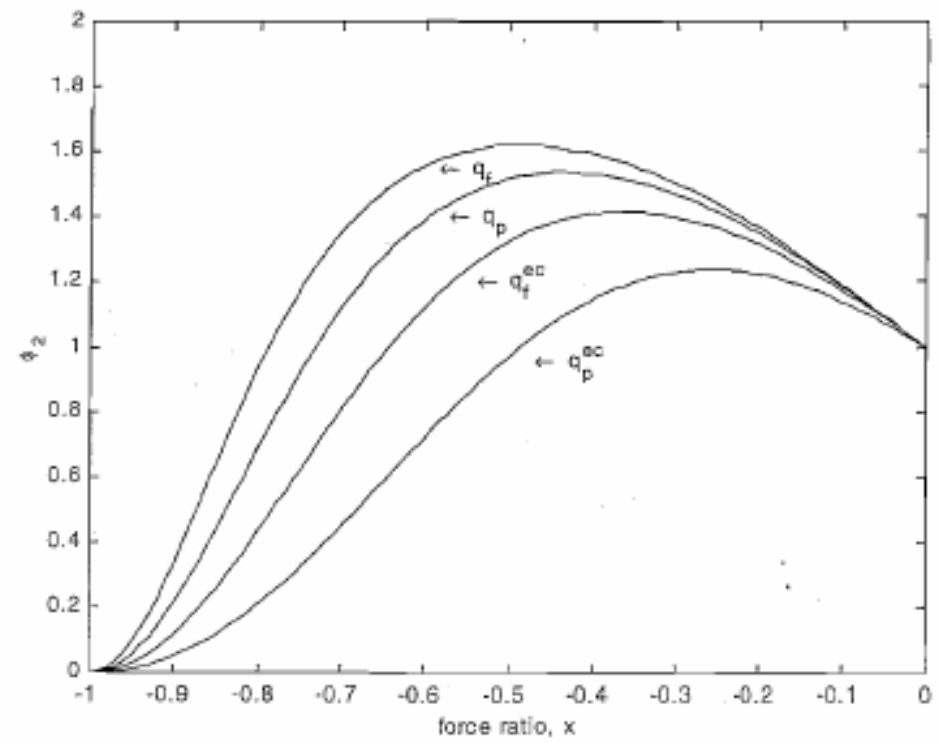


Figure 4 The change of ratio $\phi_{3}$, given in Equation (28), in terms of flow ratio $x$ and for the degrees of coupling $q_{\gamma}, q_{p}, q_{f}^{t r}$, and $q_{p}^{e c}$ with load, and $\beta=1$ and $\beta=0.9$ conductance matching
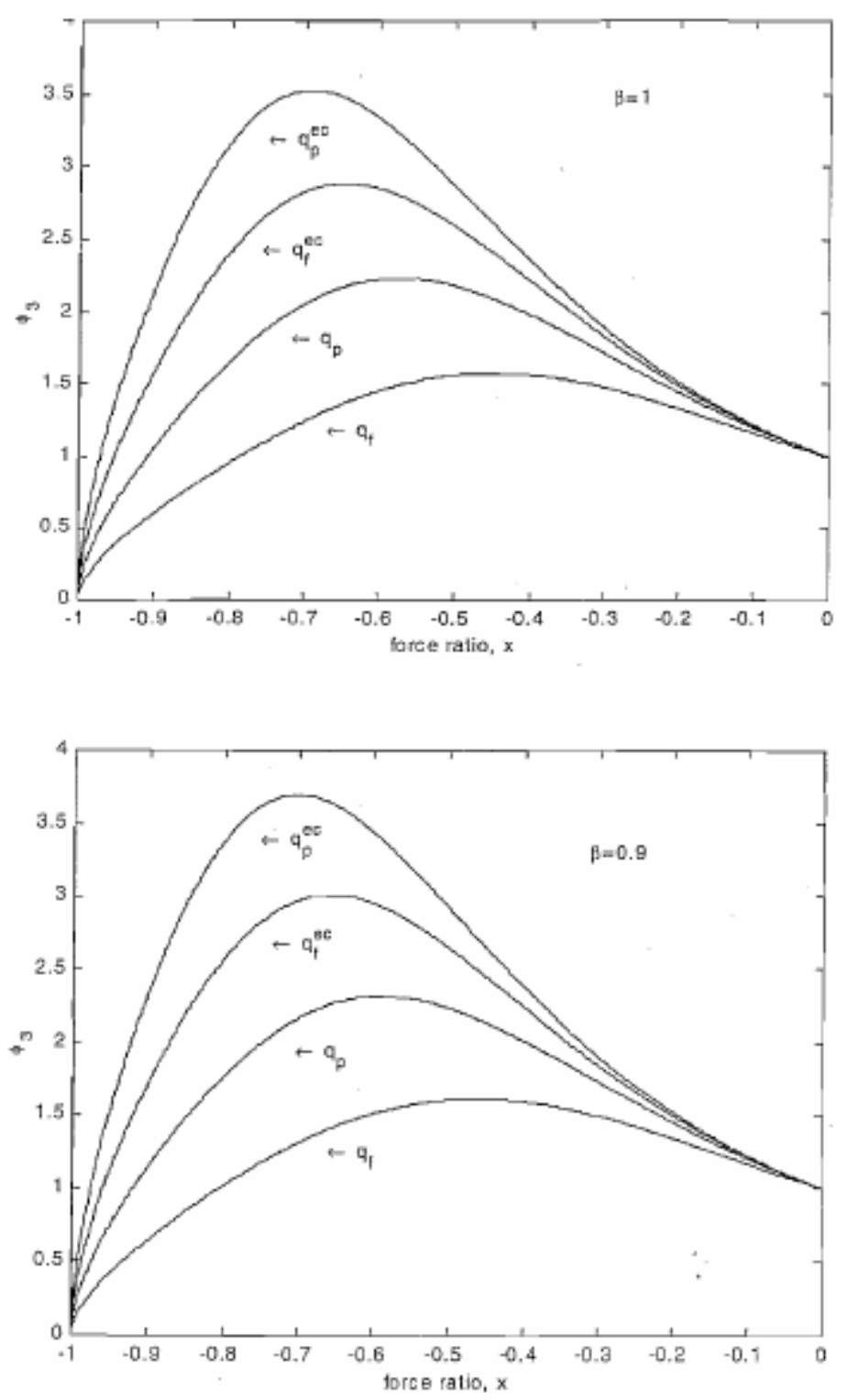
Figure 5 The change of ratio $\phi_{4}$, given in Equation (29), in terms of flow ratio $x$ and fon the degrees of coupling $q_{f}, q_{p}, q_{f}^{e c}$, and $q_{p}^{e c}$ with load and $\beta=1$ and $\beta=0.9$ conductance matching
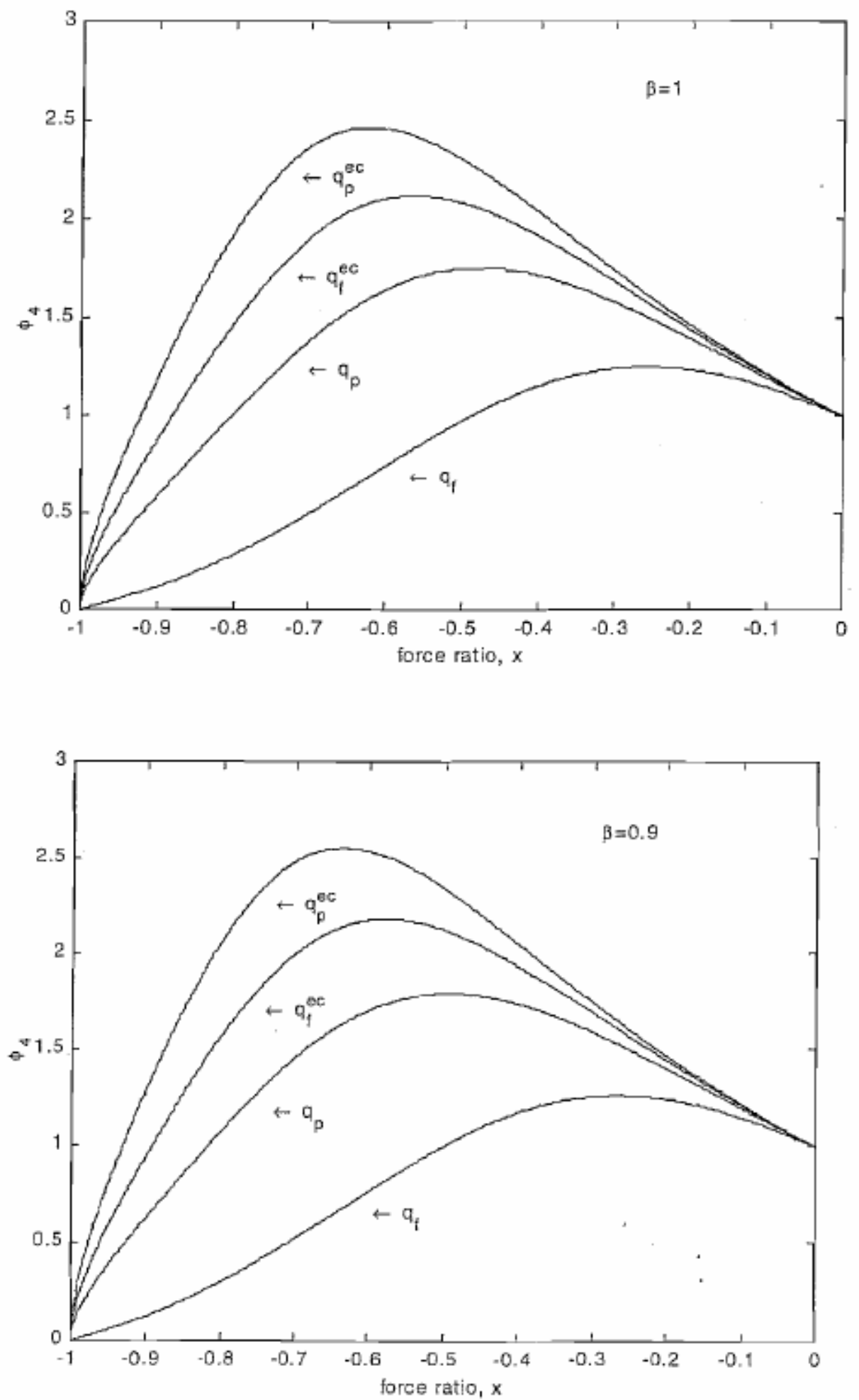
Figure 4 shows the change of $\boldsymbol{\sigma}_{3}$ expressed in Equation (28), when a load is attached with the values $\beta=$ 1 and $\beta=0.9$ at static head. With the load, the exergy destruction increases considerably. This increase is relatively larger with decreasing values of $\beta$. Figure 5 shows the values of $\boldsymbol{\emptyset}_{4}$, expressed in Equation (29), with $\beta=1$ and $\beta=0.9$. At optimal efficiency, exergy destruction is the lowest at $g_{f}>\mathbf{q}_{\mathbf{f}}$ corresponding to the maximum production of ATP, while the exergy destruction is relatively higher at economic power output with a minimal effect of $\beta$.

The load in a living cell fluctuates and compromises optimal efficiency of OP. Some enzymes operate as sensitive thermodynamic buffering to decrease deviations from optimal efficiency (Korzeniewski, 1997; Soboll, 1995; Soboll and Stucki, 1985; Stucki, 1980b; Stucki et al., 1984; Veuthey and Stucki, 1987; Westerhoff, 1993; Woitczak and Schonfeld, 1993). One intrinsic uncoupling is called the slippage, and results if one of the two coupled reactions of a cyclic process proceeds without its counterpart (Tomashek and Brusilow, 2000); for example an enzyme passes a proton without ATP synthesis, or utilizes ATP without pumping the proton. Although slippage decreases the exergetic efficiency, it enables an enzyme to control and regulate ever changing forces and demands (Tomashek and Brusilow, 2000). ATP utilizing reactions may be irreversible ones, which act as load, while reversible ones act as thermodynamic buffers. This regulatory mechanism allows OP to operate with an optimal use of the exergy contained in the nutrients. Every reversible ATP utilizing reaction can, in principle, act as a thermodynamic buffer. For example adenylate kinase can buffer the phosphate potential $X$, to the value permitting optimal efficiency of OP in the presence of too high loads (Stucki, 1980b; Stucki et al., 1984; Veuthey and Stucki, 1987). The adenylate kinase reaction is reversible, and acts as a buffer (Stucki, 1980b). If this buffer is treated as another load with a conductance $L o$, the overall load conductance $\mathrm{L}^{*}$ becomes

$$
L^{*}=L_{q} \theta+L
$$

where

$$
\begin{aligned}
\theta & =\frac{\alpha+R T \ln \left(1+\exp -\frac{\delta+X_{p}}{R T}\right)}{X_{p}}-1 \\
\alpha & =\Delta G_{a k}^{o}-R T \ln [\mathrm{M} /(1-\mathrm{M})]-\delta, \\
\mathrm{M} & =[\mathrm{AMP}] /([\mathrm{ATP}]+[\mathrm{ADP}]+[\mathrm{AMP}]), \\
\delta & =\Delta G_{p}^{o}-R T \ln [\mathrm{Pi}] .
\end{aligned}
$$

Stucki (1980b) expressed the dissipation function with buffering from Equation (23) in terms of $\mathrm{L}^{*}$

$$
\left(\Psi_{c}\right)_{b}=\left(x^{2}\left(1+\frac{L_{\alpha} \theta+L}{L_{p}}\right)+2 q x+1\right) L_{0} X_{o}^{2} .
$$

Dividing Equation (35) with Equation (23) shows file effect of thermodynamic buffering on the exergy destruction

$$
\phi_{5}=\frac{\left(\Psi_{t}\right)_{b}}{\Psi_{c}} .
$$


Figure 6 shows the change of $\varnothing_{5}$ in terms of $\mathrm{x}$ between 0 and -1 for the degrees of coupling $q_{f}, q_{p}, q_{f}{ }^{\mathrm{ec}}$ and $q_{p}{ }^{e c}$ with the load conductance of $\mathrm{L}=0.5$ and $\mathrm{L}=0.9$. The values of $\emptyset_{5}$ are lowest at economical power output and highest at maximum output of ATP. Therefore the exergy destruction is relatively lower in economical power output than that of maximum ATP flow.

Figure 6 The change of ratio $\phi_{5}$, given in Equation (36), in terms of the flow ratio $x$ anc for the degrees of coupling $q_{f}, q_{p}, q_{f}^{e c}$, and $q_{p}^{e c}$. The plots were normalised by $X_{p}=(x / Z) X_{0}$ with $X_{o}=209200 \mathrm{~J} / \mathrm{mol}, Z=3, \Delta G_{a k}^{o}=630 \mathrm{~J} / \mathrm{mol}, \Delta G_{p}^{o}=35561$ $\mathrm{J} / \mathrm{mol}, M=0.005, P_{\mathrm{i}}=8 \mathrm{mM}$ (Stucki, $1980 \mathrm{~b}$ ), $T=0.310 \mathrm{~K}$ with load conductat of $L=0.9$ and $L=0.5$
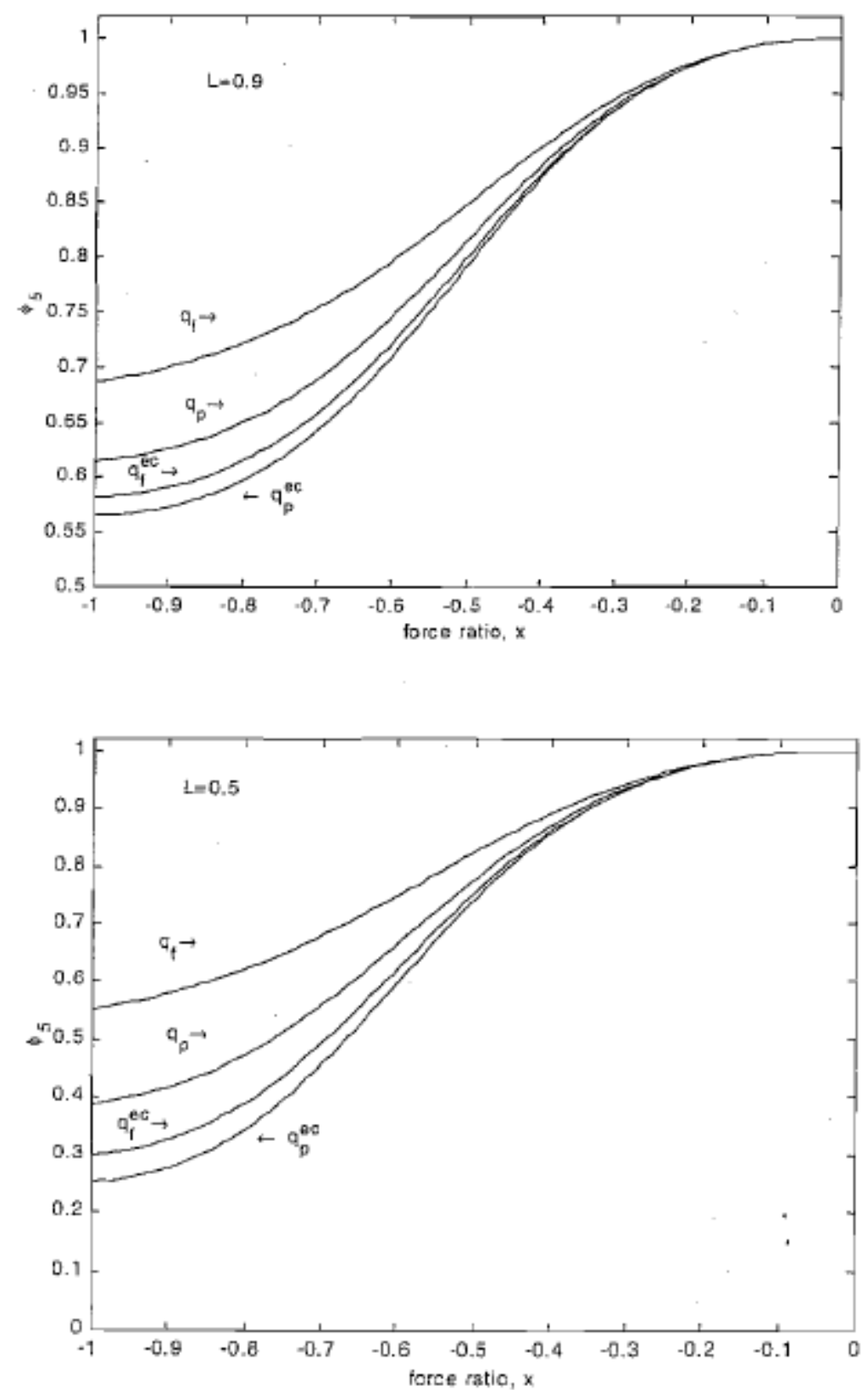

Within the framework of the theory of the dissipative structures (Kondepudi and Prigogine, 1999; Prigogine, 1967), the thermodynamic buffering (Stucki, 1980b) represents a new bioenergetics regulatory principle for the maintenance of a far from equilibrium regime. Due to the ATP production in OP, the phosphate potential is shifted far from equilibrium. Since ATP drives many processes in the cell, the shift in $X_{p}$ to far from equilibrium results a shift of all the other potentials into far from equilibrium regime. 


\section{Conclusions}

Using the nonequilibrium thermodynamic analysis, we can express the exergetic efficiency, the rate of entropy production and the exergy destroyed in terms of the degree of coupling in bioenergetics. The simplified analysis presented shows that the higher exergetic efficiency may not always imply an economical operation in oxidative phosphorylation. In bioenergetics, maximum energy production and optimum operation are adjusted and optimized for fluctuations in demands of energy production and consumption through various degrees of coupling. Like in a physical system, the exergy analysis in bioenergetics helps to describe complex-coupled phenomena.

\section{Nomenclature}

$\begin{array}{lll}A & \text { Affinity } & \mathrm{J} \mathrm{mol}^{-1} \\ G & \text { Gibbs free energy } & \mathrm{J} \mathrm{mol}^{-1} \\ J_{i} & \text { Flux of component } i & \mathrm{~mol} \mathrm{~m}^{-2} \mathrm{~s}^{-1} \\ L_{i k} & \text { Phenomenological coefficient (conductance) } & \mathrm{mol}^{2} \mathrm{~m}^{-2} \mathrm{~s}^{-1} \mathrm{~J}^{-1} \\ n & \text { Mechanistic stoichiometry } & \\ q & \text { Degree of coupling, Equation (8) } & \\ R & \text { Universal gas constant } & \mathrm{J} \mathrm{mol}^{-1} \mathrm{~K}^{-1} \\ J & \text { Rate of reaction } & \mathrm{mol}^{-1} \mathrm{~s}^{-1} \\ S & \text { Entropy } & \mathrm{J} \mathrm{K}^{-1} \\ T & \text { Absolute temperature } & \mathrm{K} \\ Z & \text { Phenomenological stoichiometry } & \\ x & \text { Ratio of forces, Equation (9) } & \\ X & \text { Thermodynamic force } & \mathrm{J} \mathrm{mol} \\ & & \\ G r e e k \text { symbols } & \mathrm{J} \cdot \mathrm{mol}^{-1} \\ \alpha & \text { Parameter, Equation (34) } & \\ \beta & \text { Conductance matching percentage } & \mathrm{J} \mathrm{mol} \\ \delta & \text { Force, Equation (34) } & \\ \phi & \text { Ratio of exergy destructions }\end{array}$




$\begin{array}{lll}\gamma & \text { Measure of relative binding affinity } & \mathrm{J} \mathrm{mol}^{-1} \\ \eta & \text { Efficiency, Equation (4) } & \\ \nu & \text { Stoichiometric coefficients } & \\ \Delta & \text { Parameter, Equation (34) } & \mathrm{J} \mathrm{mol}^{-1} \\ \zeta & \text { Reduced stoichiometry } & \end{array}$

\section{Subscripts}

$\begin{array}{ll}\text { ak } & \text { Adenylate kinase } \\ b & \text { Buffer } \\ i_{s}, k & \text { Components } \\ f & \text { Force } \\ \text { I } & \text { Linear } \\ \text { If } & \text { Level flow } \\ \text { min } & \text { Minimum } \\ \text { nl } & \text { Nonlinear } \\ o & \text { Oxyger } \\ \text { opt } & \text { Optimum } \\ p & \text { Phosphate } \\ \text { sh } & \text { Static head } \\ \text { unc } & \text { Uncoupled }\end{array}$

\section{References}

Baumann, F. (1991) 'The application of linear irreversible thermodynamics to biological growth', Acta Biotech., Vol. 11, pp.3-8.

Bisch, P.M. (1993) 'Nonequilibrium thermodynamics of biological systems', Brazilian $J$. Medical Biol. Res., Vol. 26, pp.417-424.

Cairns, C.B., Walther, J., Harken, A.L. and Banerjee, A. (1998) 'Mitochondrial oxidative phosphorylation thermodynamic efficiencies reflect physiological organ roles', Am. $J$. Physiol.-Reg. Integrative Comparative Physiol., Vol. 433, pp.R1376-R1383.

Candia, O.A. and Reinach, P.S. (1982) 'Thermodynamic analysis of active sodium and potassium-transport in the frog epithelium', Am. J. Physiol., Vol. 242, pp.F690-F698.

Caplan, S.R. and Essig A. (1983) Bioenergetics and Linear Nonequilibrium Thermodynamics, The Steady State, Cambridge: Harvard University Press.

Demirel, Y. (2002) Nonequilibritan Thermodynamics: Transport and Rate Processes in Physical and Biological Processes, Amsterdam: Elsevier.

Demirel, Y. and Sandler, S.I. (2002) 'Thermodynamics and bioenergetics', Biophys. Chem., Vol. 97, pp.87-111.

Glansdorff, P. and Prigogine, I. (1971) Thermodynamic Theory of Structure, Stability and Fluctuations, New York: Wiley.

Heinrich, R. and Schuster, S. (1998) 'The modeling of metabolic systems, structure, control and optimality', BioSystems, Vol. 47, pp.6I-77. 
Howland. J.L. and Needleman, M. (2000) 'Biological energy-coupling in terms of irreversible thermodynamics', Biochem. Mol. Biol. Edu., Vol. 28, pp.301-303.

Jencks, W.P. (1989) 'Utilization of binding energy and coupling rules for active transport and other coupled vectorial processes', Methods in Enzymology, Vol. 171, pp.145-165.

Kadenbach, B., Huttemann, M., Arnold, S., Lee, I. and Bender, E. (2000) 'Mitochondrial energy metabolism is regulated via nuclear coded subunits of cytochrome C oxidase', Free Radical Biol. and Medicine, Vol. 29, pp.211-221.

Katchalsky, A. and Curran, P.F. (1967) Nonequilibrium Thermodynamics in Biophysics, Cambridge: Harvard University Press.

Kedem, O., and Caplan, S.R. (1965) 'Degree of coupling and its relation to efficiency in energy conversion', Trans. Faraday Soc., Vol. 61, p.1897.

Kondepudi, D. and Prigogine, I. (1999) Modern Thermodynamics, From Heat Engines to Dissipative Structures, New York: Wiley.

Korzeniewski, B. (1997) 'Thermodynamic regulation of cytochrome oxidase', Mol. Cell. Biochem., Vol. 174, pp.137-141.

Krupka, R.M. (1998) 'Channelling free energy into work in biological processes', Exp. Physiol., Vol. 83, pp.243-251.

Lagarde, A.E. (1976) 'A nonequilibrium thermodynamics analysis of active transport within the framework of the chemiosmotic theory', Biochim. Biophys. Acta, Vol. 426, pp.198-217.

Lemasters, J.J. (1984) 'The ATP-to-oxygen stoichiometries of oxidative-phosphorylation by rat-liver mitochondria - an analysis of ADP-induced oxygen jumps by linear nonequilibrium thermodynamics', J. Biol. Chem., Vol. 259, pp.3123-3130.

Levenspiel, O. (1999) Chemical Reaction Engineering, New York: Wiley.

Mannella, C.A. (2000) 'Introduction: our changing views of mitochondria', J. Bioenergetics Biomemb., Vol. 32, pp.1-4.

Nath, S. (1998) 'A thermodynamic principle for the coupled bioenergetic processes of ATP synthesis', Pure Appl. Chem., Vol. 70, pp.639-644.

Nicolis, G. and Prigogine, I. (1977) Self-Organization in Noneguilibrium Systems, New York: Wiley.

Nieisen, J. (1997) 'Metabolic control analysis of biochemical pathways based on a thermokinetic description of reaction rates', Biochem. J., Vol. 321, pp.133-138.

Pfeiffer, T., Schuster, S. and Bonhoeffer, S. (2001) 'Cooperation and competition in the evolution of ATP-producing pathways', Science, Vol. 292, pp.504-507.

Prigogine, I. (1967) Introduction to Thermodynamics of Irreversible Processes, $3 \mathrm{rd} \mathrm{edn,} \mathrm{New}$ York: Wiley.

Rigoulet, M., Devin, A., Espie, P., Guerin, B., Fontaine, E., Piqiet, M-A., Nogueira, V. and Lverve, X. (1998) 'Flux-force relationships in intact cells: a helpful tool for understanding the mechanism of oxidative phosphorylation alterations?', Biochim. Biophys. Acta, Vol. 1365 , pp. $117-124$.

Rothschild, K.J., Ellias, S.A., Essig, A. and Stanley, H.E. (1980) 'Non-equilibrium linear behaviour of biological systems-existence of enzyme-mediated multidimensional inflection points', Biophys. J., Vol. 30, pp.209-239.

Rottenberg, H. (1979) 'Nonequilibrium thermodynamics of energy conversion in bioenergetics', Biochim. Biophys. Acta, Vol. 549, pp.225-253.

Rottengberg, H. and Gutman, M. (1977) 'Control of the rate of reverse electron transport in submitochondrial particles by the free energy', Biochemistry, Vol. 16, pp.3220-3227.

Santillan, M., AriasHernandez, L.A. and AnguloBrown, F. (1997) 'Some optimization criteria for biological systems in linear irreversible thermodynamics', IL Nuovo Cimento, Vol. 19, pp.99-109. 
Skulachev, V.P. (1998) 'Uncoupling: new approaches to an old problem of bioenergetics', Biochim. Biophys. Acta, Vol. 1363, pp.100-124.

Soboll, S. (1995) 'Regulation of energy metabolism in liver', J. Bioenerg. Biomemb., Vol. 27, pp. $571-582$.

Soboll, S. and Stucki, J.W. (1985) 'Regulation of the degree of coupling of oxidative phosphorylation in intact rat-liver', Biochim. Biophys Acta, Vol. 807, pp.245-254.

Stucki, J.W. (1980a) "The optimal efficiency and the economic degrees of coupling of oxidative phosphorylation', European Journal of Biochemistry, Vol. 109, pp.269-283.

Stucki, J.W. (1980b) 'The thermodynamic-buffer enzymes', Euro. J. Biochem., Vol. 109, pp.257-267.

Stucki, J.W. (1984) 'Optimization of mitochondrial energy conversions', Adv. Chemical Physic, Vol. 55, pp.141-167.

Stucki, J.W. (1991) 'Nonequilibrium thermodynamic sensitivity of oxidative phosphorylation', Proc. Royal Soc. London Series-Biol. Sci., Vol. 244, pp.197-202.

Stucki, J.W., Compiani, M. and Caplan, S.R. (1983) 'Efficiency of energy-conversion in model biological pumps optimization by linear nonequilibrium thermodynamics relation', Biophys. Chem., Vol. 18, pp.101-109.

Stucki, J.W., Lehmann, L.H. and Mani, P. (1984) 'Transient kinetics of thermodynamic buffering', Biophys. Chem., Vol. 19, pp.131-145.

Tomashek J.J. and Brusilow, W.S.A. (2000) 'Stoichiometry of energy coupling by proton-translocating ATPases: a history of variability', J. Bioenergetics Biomemb., Vol. 32, pp.493-500.

Toussaint, O. and Schneider, E.D. (1998) 'The thermodynamics and evolution of complexity in biological systems', Comparative Biochem. Physiol. A, Vol. 120, pp.3-9.

Veuthey, A-L. and Stucki, J.W. (1987) 'The adenylate kinase reaction acts as a frequency filter towards fluctuations of ATP utilization in the cell', Biophys. Chem., Vol. 26, pp.19-28.

Waldeck, A.R., Van Deam, K., Berden, J. and Kuchel, P.W. (1998) 'A non-equilibrium thermodynamics model of reconstituted Ca2+-ATPase', Euro. Biophys. J. Biophys. Let., Vol. 27, pp.255-262.

Walz, D. (1979) 'Thermodynamics of oxidation-reduction reactions and its application to bioenergetics', Biochim. Biophys. Acta, Vol. 505, pp.279-353.

Walz, D. and Caplan, S.R. (1995) 'Chemical oscillations arise solely from kinetic nonlinearity and hence can occur near equilibrium', Biophys. J., Vol. 69, pp.1698-1707.

Westerhoff, H.V. (1982) 'Should irreversible thermodynamics be applied to metabolic systems - yes - kinetics alone are impracticable', Trends in Biochem. Sci, Vol. 7, pp.275-279.

Westerhoff, H.V. (1993) 'Nonequilibrium thermodynamic consideration of the efficiency, control, and regulation of microbial-growth', Pure Appl. Chem., Vol. 65, pp.1899-1906.

Woitczak, L. and Schonfeld, P. (1993) 'Effect of fatty-acids on energy coupling processes in mitochondria', Biochim. Biophys. Acta, Vol. 1183, pp.41-57. 